\title{
Role of phospholipase $D$ in the lifespan of Caenorhabditis elegans
}

\author{
Jeong-Hwan Park', Jeong-Woo Park', Ju-Hyeon Lee ${ }^{1}$, Dong-Yun Kim², Jeong-Hoon Hahm and Young-Seuk Bae ${ }^{1,2}$
}

\begin{abstract}
We have previously shown that phospholipase D (PLD) downregulation accelerates cellular senescence, which is widely believed to play an important role in aging, by stimulating reactive oxygen species (ROS) accumulation in human cells. In this study, we examined the role of PLD in aging using the nematode Caenorhabditis elegans. The mRNA level of pld-1 was found to be inversely correlated with aging. RNAi-mediated knockdown of pld-1 expression in nematodes enhanced ROS and lipofuscin accumulation and decreased lifespan, motility, and resistance to stress compared to that in nematodes treated with control RNAi. Pld-1 knockdown repressed the long lifespan of age-1 and akt-1 mutants but did not further reduce the short lifespan of daf-16 mutants, suggesting that PLD functions between AKT-1 and DAF-16. The ROS scavenger N-acetyl-L-cysteine, a PLD effector phosphatidic acid and a possible CK2 activator spermidine attenuated the lifespan shortening and age-related biomarkers triggered by pld-1 knockdown. PId-1 RNAi downregulated the expression of DAF-16 target genes such as sod-3, dod-11, and mtl-1 in nematodes. In human cells, furthermore, PLD2 downregulation decreased the transcription of FoxO3a target genes (Cu/ZnSOD, MnSOD, catalase, thioredoxin-2, and peroxiredoxin-5), whereas ectopic PLD2 expression elevated the mRNA levels of these antioxidant genes. Taken together, these results indicated that PLD downregulation shortens longevity and induces age-related biomarkers through ROS accumulation by inhibiting the DAF-16/FoxO3a pathway in nematodes.
\end{abstract}

\section{Introduction}

The phospholipase D (PLD) lipid-signaling enzyme superfamily hydrolyzes phosphatidylcholine to generate phosphatidic acid and free choline in bacteria and eukaryotes. Phosphatidic acid plays essential roles in cellular function and contributes to membrane vesicle trafficking, anti-apoptotic signaling, malignant transformation, invasiveness, cytoskeletal reorganization, and mitogenesis as a second messenger ${ }^{1,2}$. PLD activity increases in response to mitogenic signals and is involved in cell proliferation and cancer $^{3,4}$. There are five isoforms of PLD in mammalian

\footnotetext{
Correspondence: Y-S. Bae (ysbae@knu.ac.kr)

${ }^{1}$ School of Life Sciences, BK21 Plus KNU Creative BioResearch Group, College of Natural Sciences, Kyungpook National University, Daegu 41566, Republic of Korea

${ }^{2}$ School of Life Sciences, College of Natural Sciences, Kyungpook National University, Daegu 41566, Republic of Korea

Full list of author information is available at the end of the article

These authors contributed equally: Jeong-Hwan Park, Jeong-Woo Park.
}

cells: PLD1 and PLD2 in the cytoplasm, PLD3 and PLD4 in the endoplasmic reticulum, and PLD6 in mitochondria ${ }^{1}$. However, only one PLD gene (pld-1) has been reported in the nematode Caenorhabditis elegans, which codes for a protein with 1427 amino acids ${ }^{5}$. The nematode PLD-1 protein is plentiful in neurons and pharyngeal muscles, but is present to a lesser extent in muscle and epithelial cells. Viable progeny with no apparent phenotype can be produced by pld-1 knockdown in nematodes, but the detailed impact of pld-1 knockout has not been determined ${ }^{6,7}$.

Aging is divided into intrinsic aging, which is genetically programmed, and extrinsic aging, which occurs due to exposure to environmental factors. The insulin/insulinlike growth factor (IGF)-1 signaling (IIS) pathway is a well-known pathway that controls nematode longevity. Daf-2/IGF receptor (IGFR), Age-1/phosphoinositide 3-kinase (PI3K), and Akt-1/AKT-1/2 are components of the IIS pathway ${ }^{8,9}$. The transcription factor DAF-16/ 
FoxO, which stimulates the expression of pro-longevity genes such as thermotolerant and antioxidant genes, functions downstream of the IIS pathway ${ }^{10,11}$. However, the molecular mechanism by which this signaling pathway regulates C. elegans longevity remains to be elucidated.

It has been reported that PLD activity is decreased in senescent cells ${ }^{12,13}$. We have previously shown that PLD transcription decreases during both replicative and premature senescence in human diploid fibroblast IMR-90 and colon cancer HCT116 cells. Knockdown of PLD2 causes premature senescence via the $\mathrm{p} 53-\mathrm{p} 21^{\mathrm{Cip} 1 / \mathrm{WAF} 1}$ pathway by stimulating reactive oxygen species (ROS) accumulation in cells ${ }^{14}$. In this study, we investigated the physiological significance of PLD in nematode aging. Our results indicated that pld-1 downregulation caused ROS accumulation, decreased longevity, and induced age-related biomarkers. Treatment with the ROS scavenger $\mathrm{N}$-acetyl-L-cysteine (NAC), a putative CK2 activator spermidine, and a PLD effector phosphatidic acid, attenuated pld-1 RNAi-mediated lifespan shortening. PLD downregulation reduced the expression of DAF-16/FoxO target genes such as superoxide dismutase (SOD). The present study suggests that PLD plays a critical role in healthy lifespan via a connection to DAF-16/FoxO-mediated expression of antioxidant proteins.

\section{Materials and methods}

\section{Culture of nematode strains}

Nematode N2 (wild-type) strain, strains carrying mutant alleles daf-16(mu86), age-1(hx546), and akt-1 (mg144), and reporter strains sod-3::gfp, dod-11::gfp, dod$8:: g f p$, and $m t l-1:: g f p$ were acquired from the Caenorhabditis Genetics Center. Nematodes were grown at $21^{\circ} \mathrm{C}$ on nematode growth medium (NGM) agar plates with Escherichia coli strain OP50 as a food source. For some experiments (Fig. 4, Supplemental Figs. 2 and 3), nematodes were treated with NAC (Sigma-Aldrich, MO), spermidine (Sigma-Aldrich, MO), or phosphatidic acid (Sigma-Aldrich, MO).

\section{RNAi experiments}

E. coli HT115 cells expressing double-stranded pld-1 RNA were obtained from the C. elegans ORFeome RNAi library. To deactivate pld-1 function, eggs from gravid adults were placed on HT115-seeded NGM plates and allowed to hatch. Expression of double-stranded RNA was induced by treating with $1 \mathrm{mM}$ isopropyl 1 -thio- $\beta$-Dgalactopyranoside. Nematodes hatched from eggs were fed on HT115-seeded NGM plates until the L4 stage. To synchronize the nematodes, L4 larvae were then placed on HT115-seeded NGM plates supplemented with $5 \mu \mathrm{M}$ 5-Fluoro-2'-deoxyuridine (FUdR; Sigma-Aldrich, MO), which prevents offspring production, and were allowed to grow to day 1 or 8 of adulthood. E. coli HT115 containing the empty L4440 vector was used as an RNAi control. For some experiments (Figs. 2b and 3b, Supplemental Figure $1 \mathrm{~b}$ ), we used nematodes fed with pld-1 RNAi at the L4 larval stage for 1 day to eliminate the effect of pld-1 downregulation on nematode development.

\section{ROS measurement}

Synchronized (day 1 and 8 of adulthood) nematodes were washed with $\mathrm{M} 9$ buffer $\left(22 \mathrm{mmol} / \mathrm{L} \mathrm{KH}_{2} \mathrm{PO}_{4}, 22\right.$ $\mathrm{mmol} / \mathrm{L} \quad \mathrm{Na}_{2} \mathrm{HPO}_{4}, \quad 85 \mathrm{mmol} / \mathrm{L} \mathrm{NaCl}$, and $1 \mathrm{mmol} / \mathrm{L}$ $\mathrm{MgSO}_{4}$ ) and then transferred to $2 \mathrm{~mL}$ of $\mathrm{M} 9$ buffer containing $6 \mu \mathrm{M}$ dichlorofluorescein diacetate (DCFDA, Invitrogen, $\mathrm{CA}$ ) or $3 \mu \mathrm{M}$ dihydroethidium (DHE, Invitrogen, CA). After incubation for $60 \mathrm{~min}$ at $37^{\circ} \mathrm{C}$ in the dark, the nematodes were rinsed with M9 buffer, and digital images were obtained with a Leica DM IRB inverted microscope (Leica Microsystems, Germany) equipped with a CoolSNAP HQ camera (Roper Scientific, NJ) operated by Metamorph Image Software (Universal Imaging Corporation, PA). Three independent experiments were performed.

\section{Lifespan assay}

Lifespan assays were performed as described previously ${ }^{15}$. Synchronized L4 larvae were placed on HT115-seeded NGM plates containing FUdR. Surviving nematodes were counted daily and were moved to fresh HT115-seeded NGM plates. Death was scored as the absence of a response to slight touch using a thin platinum wire. Three independent experiments were performed.

\section{Locomotion velocity assays}

The nematodes' mean velocity and maximum velocity were measured as previously described ${ }^{16}$. Briefly, synchronized (day 1 and 8 of adulthood) nematodes were transferred to an NGM plate without a bacterial lawn, and their movements were immediately recorded. The recording system contained a stereomicroscope (Nikon SMZ 745T), a charge-coupled device camera (TUCSEN TCH-5.0), and imaging software (TUCSEN ISCapture). The recording period was $30 \mathrm{~s}$ at a rate of 30 frames per second. The locomotion velocity was expressed as $\mathrm{mm}$ per second (the distance $(\mathrm{mm})$ between displaced centroids per second). Recorded images were evaluated by ImageJ and wrMTrck (plugin for ImageJ: www.phage.dk/plugins). The locomotion velocity data were imported into an Excel worksheet. The highest locomotion velocity observed in the 30 -s period was used as the maximum velocity.

\section{Lipofuscin assay}

Intestinal lipofuscin accumulation in synchronized (day 1 and 8 of adulthood) nematodes was determined by autofluorescence as previously described ${ }^{17}$. The 
autofluorescence of lipofuscin was assessed using a fluorescence microscope (ZEISS AxioCam MRc, Germany) with excitation and emission wavelengths of 350 and $470 \mathrm{~nm}$, respectively. The relative fluorescence intensity was quantified using ImageJ software (National Institutes of Health, MD) to determine lipofuscin levels.

\section{Assays for oxidative and heat stress resistance}

For oxidative stress resistance assays, synchronized (day 8 of adulthood) nematodes were transferred to HT115seeded NGM plates containing $5 \mathrm{mM}$ of $\mathrm{H}_{2} \mathrm{O}_{2}$. After $5 \mathrm{~h}$ of incubation, nematode survival was scored as touchprovoked movement every $4 \mathrm{~h}$. For thermotolerance assays, HT115-seeded NGM plates with synchronized (day 1 and 8 of adulthood) nematodes were shifted from $21^{\circ} \mathrm{C}$ to $35^{\circ} \mathrm{C}$. After the temperature shift, nematode viability was scored as touch-provoked movement every 2 h. Fifty nematodes were used in each experimental group. Data were analyzed and plotted as described for lifespan assays.

\section{Assays for reporter gene expression}

Synchronized (L4 larva) reporter nematodes sod-3:: gfp, dod-11::rfp, mtl-1::rfp, and dod-8::gfp were transferred to HT115-seeded NGM plates. After 1 day, the fluorescence of GFP (green fluorescent protein) or RFP (red fluorescent protein) was measured using a fluorescence microscope (ZEISS AxioCam MRc, Germany) at excitation and emission wavelengths of $490 \mathrm{~nm}$ and $525 \mathrm{~nm}$, respectively. The relative fluorescence intensity was quantified using ImageJ software to determine GFP levels. Three independent experiments were performed.

\section{Reverse transcription-PCR (RT-PCR)}

Total RNA was extracted fromsynchronized (day 1 or 12 of adulthood) nematodes or human HCT116 and MCF-7 cells. RNA was reverse-transcribed using genespecific reverse primers and reverse transcriptase (Solgent, Korea), and the resulting cDNA was PCR-amplified. Primers used for assays are listed in Supplementary Table 1. Primers for act- 2 and $\beta$-actin were used to normalize to RNA concentration in each sample. PCR products were resolved on a 1.5\% agarose gel. Quantification of RT-PCR bands was performed using densitometry.

\section{Statistical analysis}

The statistical significance of data was analyzed by one-way ANOVA with the SPSS package program. The results were considered significant if the $P$ value was less than 0.05. Duncan's multiple-range test was performed if differences between groups were identified as $\alpha=0.05$.

\section{Results}

Expression level of pld-1 inversely correlates with aging and pld-1 RNAi promotes ROS accumulation in nematodes

We previously showed that PLD is transcriptionally repressed during senescence in human cells and PLD2 inhibition stimulates ROS formation in human cells ${ }^{14}$. To investigate whether PLD downregulation increases ROS levels in nematodes, we compared ROS levels in nematodes treated with pld-1 RNAi to those in nematodes treated with control (L4440) RNAi. The mRNA level of pld-1 was reduced in nematodes treated with pld-1 RNAi, relative to that of nematodes treated with control RNAi (Fig. 1a). ROS quantification by DCFDA or DHE staining demonstrated that ROS levels apparently increased in pld-1-knockdown nematodes as compared to those in control nematodes. Consistently, an apparently higher level of ROS was observed in wild-type nematodes at day 8 of adulthood compared to wild-type nematodes at day 1 of adulthood (Fig. 1b, c). We next investigated whether the expression level of pld-1 was related to aging in nematodes. The mRNA level of pld-1 was decreased by $70 \%$ in nematodes at day 12 compared to that in nematodes at day 1 (Fig. 1d).

\section{PId-1 RNAi decreases lifespan and resistance to oxidative and heat stress in nematodes}

We previously reported that PLD2 knockdown caused cellular senescence in human cells ${ }^{14}$. To determine if PLD knockdown reduced nematode lifespan, we determined the lifespan of pld-1 RNAi nematodes and control nematodes. After pld-1 RNAi treatment, the maximum lifespan of the nematodes was shortened from 24 to 18 days, and the median lifespan decreased from 18 to 12 days (Fig. 2a). To eliminate the effect of pld-1 knockdown on nematode development, we performed lifespan assays using nematodes fed pld-1 RNAi from the L4 larval stage and obtained similar results (Fig. 2b). Aged nematodes display reduced resistance to oxidative stress and heat stress ${ }^{8}$. We thus analyzed the impact of pld-1 knockdown on resistance to oxidative stress $\left(5 \mathrm{mM} \mathrm{H}_{2} \mathrm{O}_{2}\right)$ and heat stress $\left(35^{\circ} \mathrm{C}\right)$ in nematodes. As shown in Fig. 2c, d, pld-1 RNAi suppressed resistance to oxidative stress and heat stress. Collectively, these data indicate that proper PLD activity is important for longevity and stress resistance in nematodes.

\section{PId-1 RNAi increases lipofuscin accumulation and reduces physical velocity in nematodes}

Lipofuscin accumulation and decreased motility are characteristics of nematode aging ${ }^{8,17}$. To analyze the impact of pld-1 on lipofuscin amount in C. elegans, lipofuscin levels were measured by autofluorescence. More lipofuscin was present in pld-1 RNAi nematodes relative to levels in control RNAi nematodes (Fig. 3a and Supplemental Figure 1a). To eliminate the effect of pld-1 

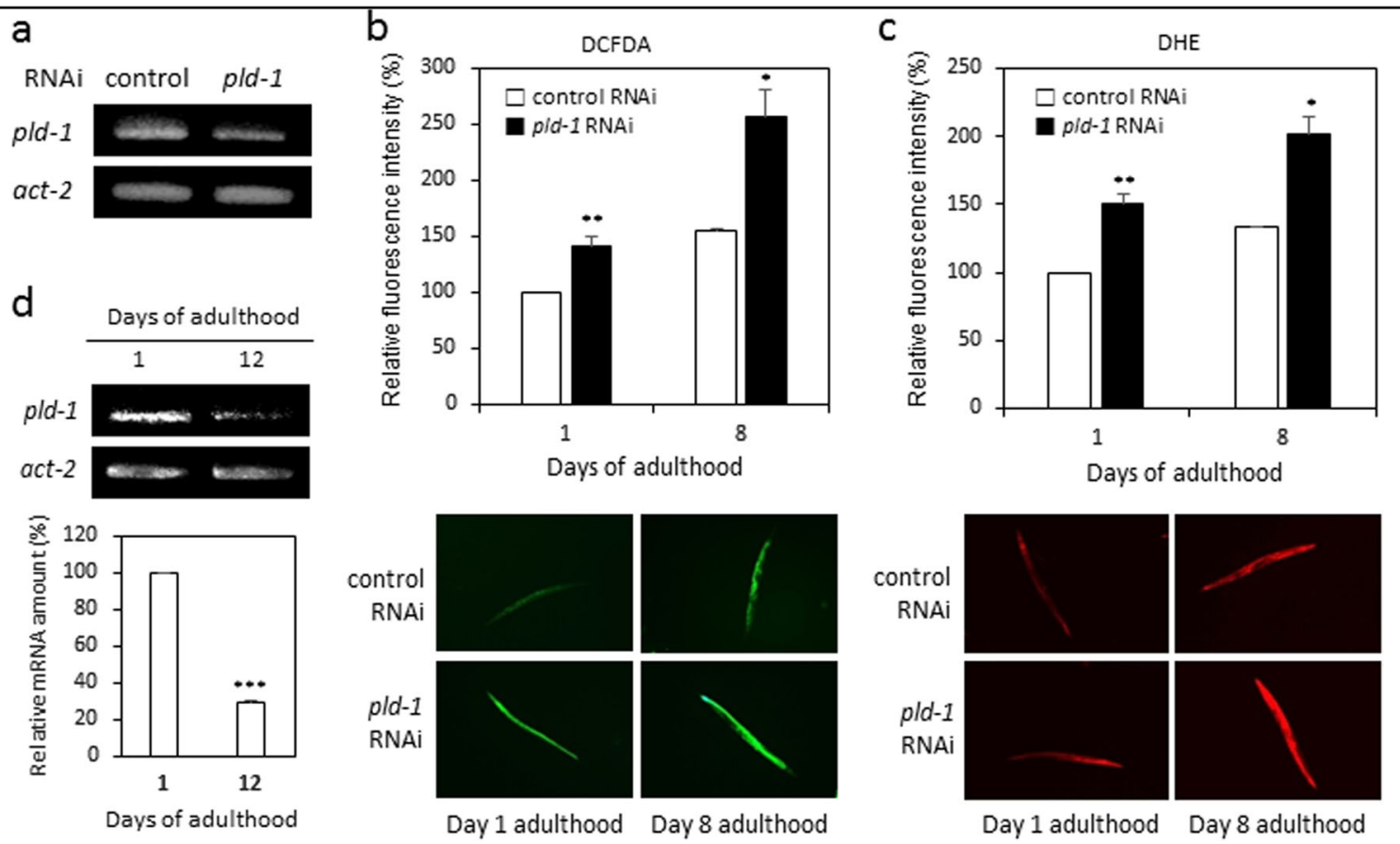

Fig. 1 PId-1 RNAi promotes ROS formation in nematodes and pld-1 expression inversely correlates with aging. a Effect of pld-1 RNAi on pld-1 expression in nematodes. Lysates from nematodes at day 1 of adulthood fed with pld-1 RNAi or control RNAi were utilized in RT-PCR using specific pld-1 primers. PCR products were resolved on a 1.5\% agarose gel. Representative data from three independent experiments are shown. act-2 mRNA served as the loading control. Nematodes fed with pld-1 RNAi or control RNAi were incubated with DCFDA (b) or DHE (c). The fluorescence intensity of nematodes at day 1 or 8 of adulthood (upper panels) was quantified using ImageJ software by determining the average pixel intensity $(n>116$ per day 1 condition; $n>73$ per day 8 condition). Data are shown as the means \pm SEM. ${ }^{*} P<0.05,{ }^{* *} P<0.01$. Representative images of nematodes were obtained at 10x magnification (bottom panels). d Age-dependent reduction in pld-1 mRNA levels. Lysates from nematodes at days 1 and 12 of adulthood were utilized in RT-PCR using specific pld-1 primers. PCR products were resolved on a 1.5\% agarose gel (upper panel). Representative data from three independent experiments are shown. act-2 mRNA served as the loading control. The graph shows the relative mRNA level of pld-1 (lower panel). Data are shown as the means \pm SEM. ${ }^{* * *} P<0.001$

knockdown on nematode development, we investigated lipofuscin accumulation in nematodes fed pld-1 RNAi at the L4 larval stage for 1 day and obtained similar results (Fig. 3b and Supplemental Figure 1b). We next determined nematode motility. Pld-1 knockdown apparently reduced the average velocity (Fig. 3c) and maximum velocity (Fig. 3d) of nematodes. Therefore, these results suggest that a proper amount of PLD is required for healthy lifespan and motility in nematodes.

\section{A ROS scavenger NAC, a possible CK2 activator} spermidine, and a PLD effector phosphatidic acid rescue the lifespan shortening and age-related biomarkers mediated by pld-1 knockdown in nematodes

To analyze the role of ROS in pld-1 knockdown-mediated lifespan shortening, nematodes were incubated with NAC, a commonly used ROS scavenger. To determine whether NAC attenuates lifespan shortening upon pld-1 knockdown, pld-1-deficient nematodes were exposed to NAC. Treatment with NAC (6 or $9 \mu \mathrm{M})$ counteracted the lifespan shortening mediated by pld-1 knockdown (Fig. 4a and
Supplemental Fig. 2). These findings indicate that in worms, ROS formation is one of the major upstream causes of aging triggered by PLD downregulation.

We previously reported that ectopic CK2 $\alpha$ expression stimulates PLD2 activity and represses PLD2 knockdownmediated senescence in human cells ${ }^{14,18}$. Polyamines such as spermine, spermidine, and polylysine have been known to stimulate CK2 activity in vitro ${ }^{19}$. Expression of ornithine decarboxylase, which catalyzes the synthesis of a polyamine precursor molecule, stimulates CK2 activity in vivo ${ }^{20}$. To examine whether enhanced CK2 activity abrogated the lifetime shortening induced by pld1 knockdown, nematodes were incubated with spermidine. Treatment with spermidine $(0.2 \mu \mathrm{M})$ apparently counteracted the lifespan shortening mediated by pld-1 knockdown. Furthermore, treatment with spermidine $(0.2 \mu \mathrm{M})$ significantly extended lifespan in wild-type nematodes: the maximum and median lifespans were extended from 26 to 32 days and from 20 to 22.5 days, respectively (Fig. 4a and Supplemental Fig. 2).

PLD catalyzes the hydrolysis of phosphatidylcholine into choline and phosphatidic acid, which functions as an 

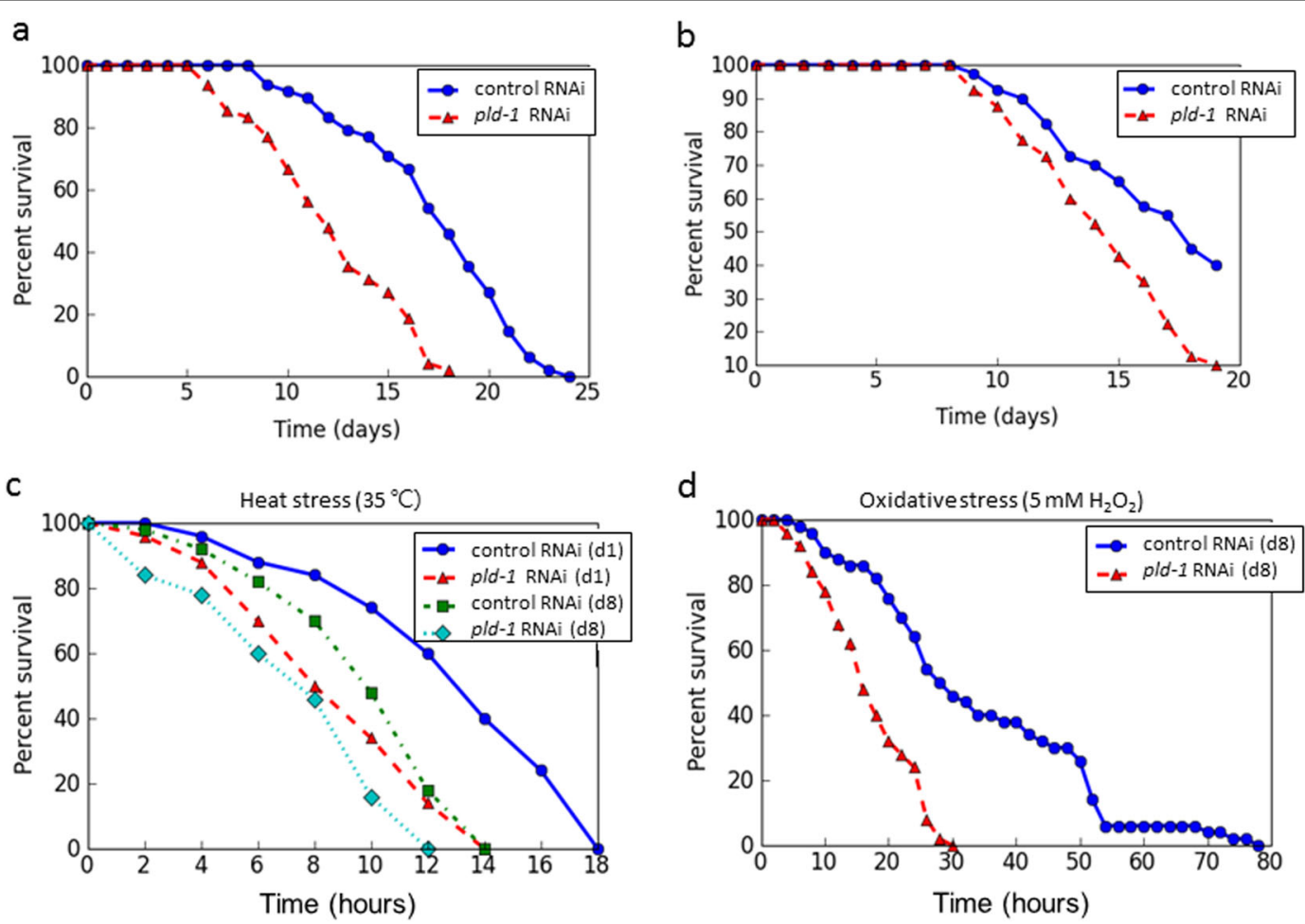

Fig. 2 PId-1 RNAi decreases lifespan and stress resistance in nematodes. $\mathbf{a}$, $\mathbf{b}$ Effect of pld-1 RNAi on the lifespan of nematodes. a Agesynchronized L4 larvae were fed on control RNAi or pld-1 RNAi plates under standard conditions ( $n=50$ per condition). b Lifespan assays using nematodes fed with pld-1 RNAi only during adulthood to exclude the effect of pld-1 knockdown on development. Age-synchronized L4 larvae were fed on control RNAi or pld-1 RNAi plates for 1 day ( $n=50$ per condition). $\mathbf{c}$, $\mathbf{d}$ Effect of pld-1 RNAi on stress resistance in nematodes. $\mathbf{c}$ Survival curves of nematodes fed with control or pld-1 RNAi at $35^{\circ} \mathrm{C}$. Nematodes at day 1 (d1) or 8 (d8) of adulthood were scored every $2 \mathrm{~h}$ for viability $(n=50$ per condition). $\mathbf{d}$ Survival curves of nematodes fed with control or pld-1 RNAi after exposure to $5 \mathrm{mM} \mathrm{H}_{2} \mathrm{O}_{2}$ for 5 h. Nematodes at day 8 (d8) of adulthood were scored every $4 \mathrm{~h}$ for viability ( $n=50$ per condition). Viability was scored as movement away from pick touch at the indicated days or hours. Representative data from three independent RNAi experiments are shown

effector in multiple signaling pathways ${ }^{1-4}$. We investigated the impact of phosphatidic acid on nematode longevity. Treatment with phosphatidic acid $(30 \mu \mathrm{M})$ successfully counteracted the shortened lifespan mediated by pld-1 knockdown. Furthermore, treatment with phosphatidic acid $(30 \mu \mathrm{M})$ significantly extended the lifespan of wild-type nematodes, suggesting that PLD-1 plays an important role in C. elegans longevity. After phosphatidic acid $(30 \mu \mathrm{M})$ treatment, the maximum and median lifespan of the wild-type nematodes was extended from 26 to 35 days and from 20 to 28 days, respectively (Fig. 4a and Supplemental Fig. 2).

Furthermore, treatment with NAC, spermidine, or phosphatidic acid significantly attenuated lipofuscin accumulation (Fig. 4b and Supplemental Fig. 3a), decreased motility (Fig. 4c and Supplemental Fig. 3b), and susceptibility to heat and oxidative stress (Fig. 4d, e) mediated by pld-1 knockdown. Consistently, treatment with these chemicals reduced lipofuscin accumulation (Fig. 4b and Supplemental Fig. 3a) and stimulated resistance to heat and oxidative stress (Fig. 4d, e) in wild-type nematodes.
PLD modulates the transcription of DAF-16/FoxO3a target genes in nematodes and human cells

DAF-16/FoxO is a central transcription factor that detoxifies intracellular ROS by expressing antioxidant proteins ${ }^{10,11}$. To determine whether pld-1 knockdown decreased the transcriptional activity of DAF-16, we evaluated the fluorescence of several reporters that are specific for certain stress pathways, including sod-3, which encodes manganese superoxide dismutase (MnSOD); dod-11, which encodes sorbitol dehydrogenase; $m t l-1$, which encodes metallothionein; and $d o d-8$, which encodes $17 \beta$-hydroxysteroid dehydrogenase ${ }^{21}$. As shown in Fig. 5a, pld-1 knockdown suppressed the transcription of sod-3::gfp, dod-11::rfp, and mtl-1:rfp reporter genes relative to expression upon exposure to empty vector RNAi. In contrast, pld-1 knockdown stimulated transcription of the dod-8::gfp reporter gene (Fig. 5a). To examine a similar effect of PLD on transcription of FoxO3a target genes $(\mathrm{Cu} / \mathrm{ZnSOD}, \mathrm{MnSOD}$, catalase, thioredoxin-2, and peroxiredoxin-5) in human cells, PLD2 siRNA or PLD2 expression vectors were transfected 

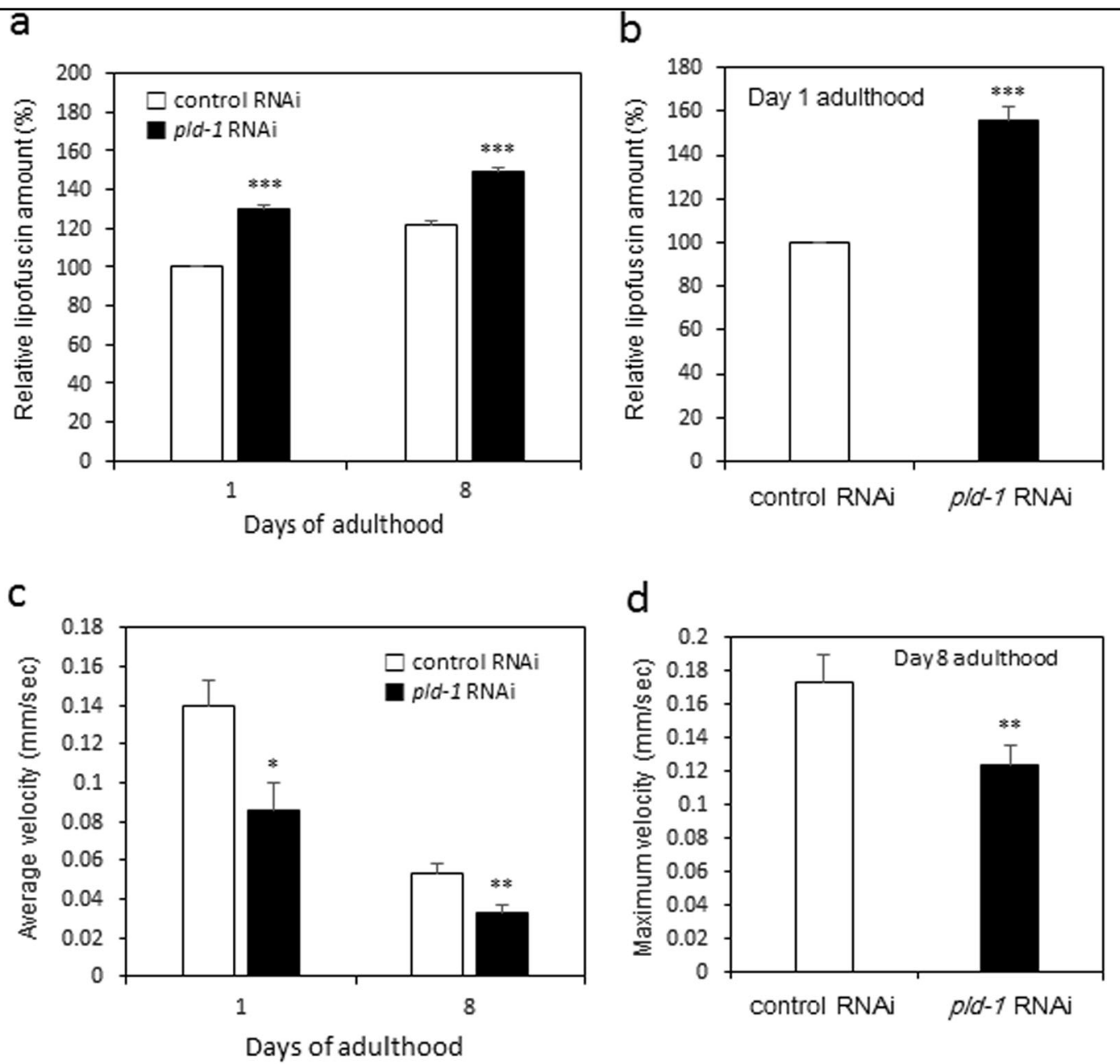

Fig. 3 PId-1 RNAi increases lipofuscin accumulation and reduces the physical velocity of nematodes. $\mathbf{a}$, $\mathbf{b}$ Effect of pld-1 RNAi on lipofuscin accumulation. a Age-synchronized L4 larvae were fed on control RNAi or pld-1 RNAi plates under standard conditions. $\mathbf{b}$ Effects of pld-1 RNAi on lipofuscin accumulation in nematodes fed pld-1 RNAi for 1 day at the L4 larval stage, to eliminate the effect of pld-1 knockdown on development. The autofluorescence images were obtained with a fluorescence microscope. The fluorescence intensity was quantified using ImageJ software by determining the average pixel intensity ( $n>134$ per day 1 condition; $n>103$ per day 8 condition). The average velocity (c) and maximum velocity (d) of individual nematodes were measured at day 1 or 8 of adulthood ( $n=20$ per condition). Data are shown as the means \pm SEM. ${ }^{*} P<0.05$; ${ }^{* *} P<0.01$; ${ }^{* * *} P<0.001$

into HCT116 and MCF-7 cells. PLD2 knockdown decreased mRNA levels of FoxO3a target genes in HCT116 cells and MCF-7 cells (Fig. 5b). Conversely, ectopic PLD2 expression increased the mRNA levels of these antioxidant genes in these cells (Fig. 5c). Thus, the present data suggested that PLD increases intracellular antioxidant levels through downregulating DAF-16/ FoxO3a activity in nematodes and human cells.

\section{Pld-1 regulates longevity between AKT-1 and DAF-16 in nematodes}

We next investigated the relationship between DAF-16 and PLD-1 in nematodes using the daf-16(mu86) mutant. As shown in Fig. 6a, the longevity of daf-16(mu86) mutant nematodes was not synergistically reduced by pld-1 RNAi treatment, suggesting that PLD-1 and DAF-16 are positioned in the same pathway for lifespan control. Because AGE-1 and AKT-1 lie upstream of DAF-16 in the IIS pathway ${ }^{8-11}$, we investigated the role of AGE-1 and AKT-1 in PLD-mediated longevity regulation using the age-1(hx546) and akt-1(mg144) single mutants. Even though the lifespans of both mutants were drastically increased compared with lifespans of control nematodes, pld-1 RNAi completely repressed the longevity extension effect of the age-1(hx546) and akt-1(mg144) mutations (Fig. 6b, c). We next examined whether the mRNA level of pld-1 was upregulated in the age-1(hx546) and akt-1 (mg144) mutant nematodes. The mRNA level of pld-1 was increased by $170 \%$ in these mutants compared to that in wild-type N2 nematodes at day 12 of adulthood (Fig. 6d). Collectively, these data suggest that AGE-1 and AKT-1 are upstream regulators of PLD in nematodes. 


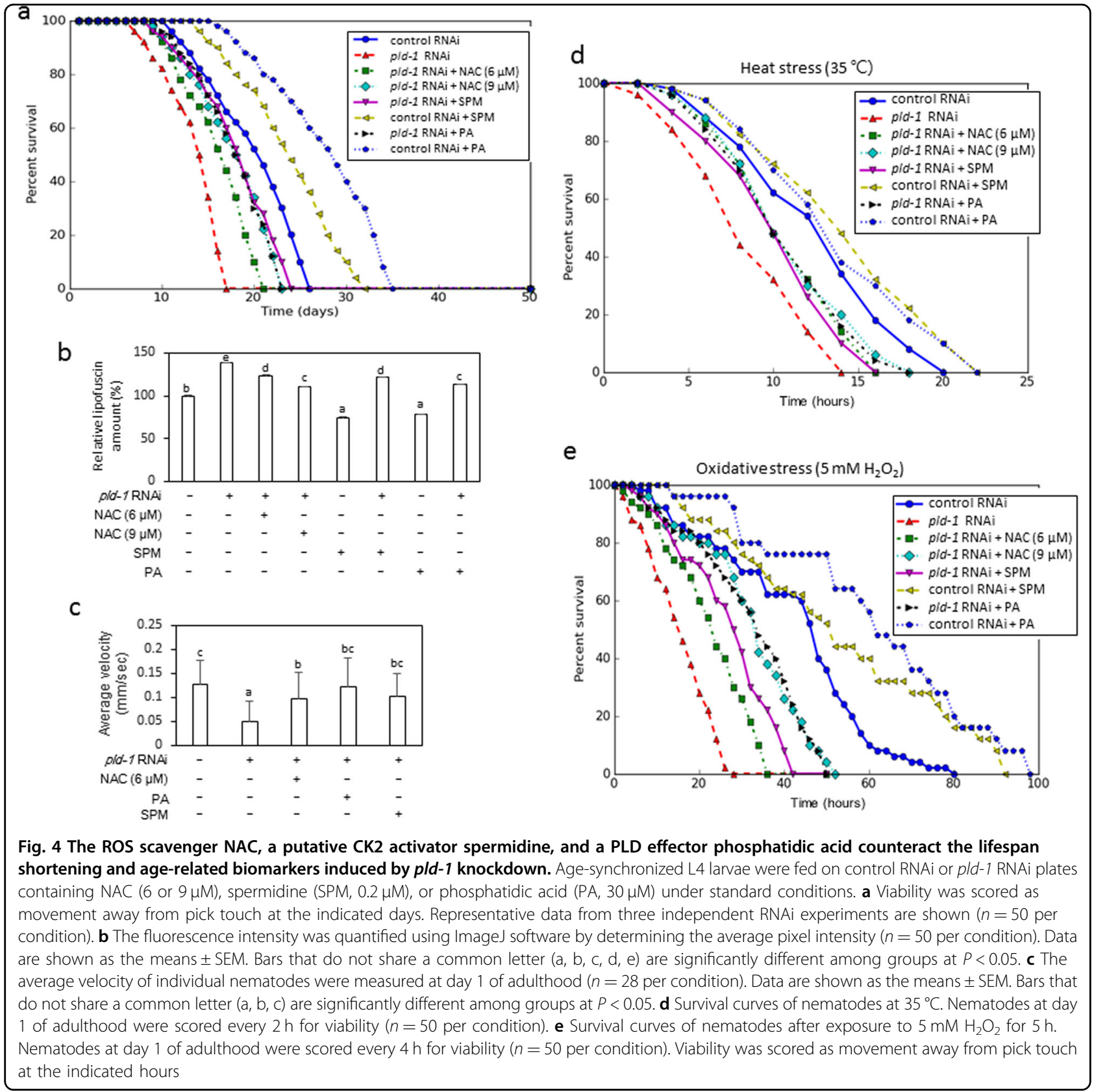

\section{Discussion}

We previously demonstrated that PLD expression decreases during replicative senescence in IMR-90 cells and PLD2 knockdown triggers premature senescence in HCT116 and MCF-7 cells, suggesting that decreased PLD activity is tightly linked to the senescence process ${ }^{14}$. Because nematodes are broadly used as an animal model for studying aging, we examined the role of pld-1 in nematode aging. The present study showed that pld-1 knockdown shortened longevity (Fig. 2a, b), reduced resistance to heat and oxidative stress (Fig. 2c, d), increased lipofuscin accumulation (Fig. 3a, b, Supplemental Fig. 1), and decreased motility (Fig. 3c, d) in nematodes. Because reduced resistance to oxidative stress and heat stress, lipofuscin accumulation, and declined velocity of body movement are distinctive features of aging ${ }^{8,16,17}$, these results collectively suggest that pld-1 knockdown causes a range of age-related biomarkers in nematodes. Consistently, phosphatidic acid, which acts as a downstream effector of PLD, successfully counteracted the pld-1 knockdown-mediated lifespan shortening and age-related biomarkers, including retardation of locomotion, increased lipofuscin accumulation, and reduced resistance to heat and oxidative stress (Fig. 4, Supplemental Figs. 2 and 3). In addition, phosphatidic acid considerably extended lifespan 
and reduced age-related biomarkers in wild-type nematodes (Fig. 4, Supplemental Figs. 2 and 3a). Notably, the mRNA level of pld-1 inversely correlated with aging in nematodes (Fig. 1d). This is consistent with results of a previous study showing that PLD is transcriptionally repressed during senescence in human cells ${ }^{14}$. Based on these data, we propose that sufficient PLD activity is necessary for extending healthy lifespan in nematodes.

Numerous reports have demonstrated that increased intracellular oxidative stress might be a critical mechanism for aging development ${ }^{22,23}$. We also previously showed that PLD2 knockdown triggers cellular senescence via ROS formation in human cells ${ }^{14}$. The present results also demonstrated that pld-1 knockdown significantly increased ROS accumulation in nematodes (Fig. 1b, c). Moreover, the antioxidant NAC apparently abrogated the longevity shortening and age-related biomarkers triggered by pld-1 knockdown, indicating that ROS is an important upstream trigger of aging induced by PLD downregulation in nematodes (Fig. 4, Supplemental
Figs. 2 and 3). We examined the role of DAF-16/FoxO3a, a major transcription factor for antioxidant genes, in PLD knockdown-mediated ROS accumulation. The present results showed that the transcriptional activity of DAF-16 on the promoters of sod-3 and $\operatorname{dod}-11$ decreased upon pld-1 knockdown in nematodes (Fig. 5a). These data suggest that PLD knockdown-mediated downregulation of SOD-3/MnSOD, which detoxifies $\mathrm{ROS}^{24}$, and DOD$11 /$ sorbitol dehydrogenase, which reduces the nicotinamide adenine dinucleotide levels in the conversion of sorbitol to fructose ${ }^{25}$, can cause ROS accumulation in nematodes. Our present experiments using human PLD2 also confirmed this phenomenon; downregulating human PLD2 decreased the transcription of antioxidant genes $(\mathrm{Cu} / \mathrm{ZnSOD}, \mathrm{MnSOD}$, catalase, thioredoxin-2, and peroxiredoxin-5) in HCT116 and MCF-7 cells, whereas ectopic PLD2 expression increased the transcription of antioxidant genes in these cells (Fig. 5b, c). The fact that the longevity of the daf-16(mu86) mutant was not synergistically reduced by pld-1 RNAi indicates that PLD-

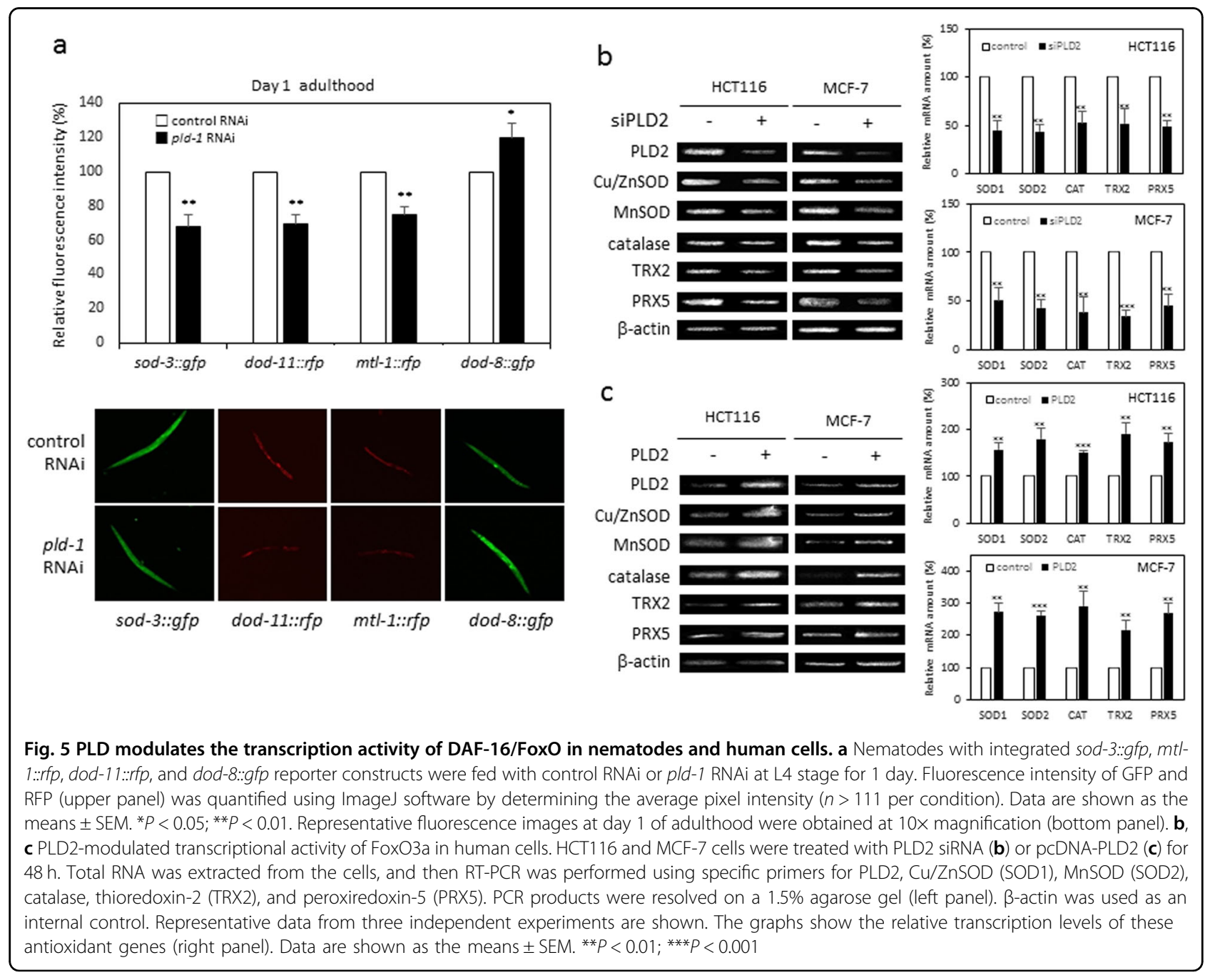




\section{a}

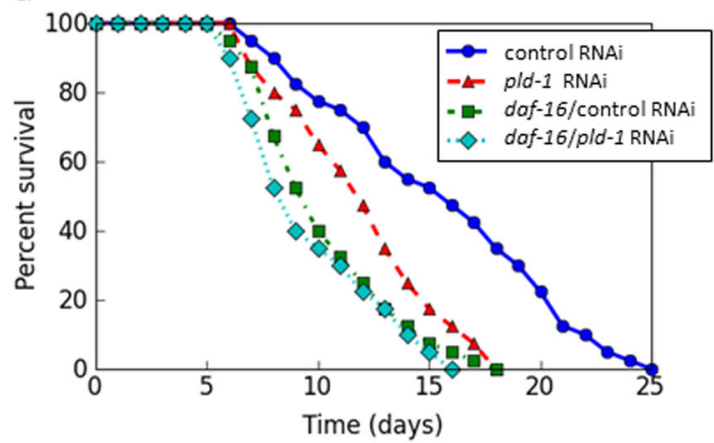

C

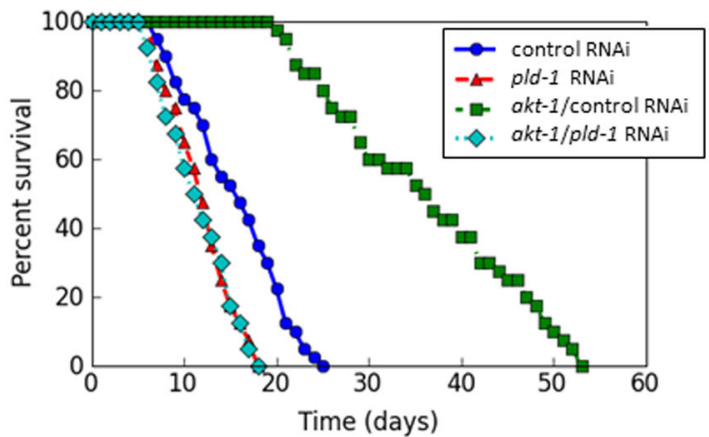

b

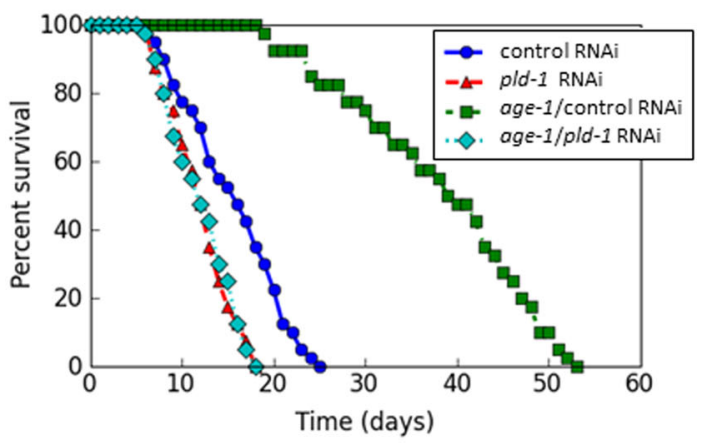

d

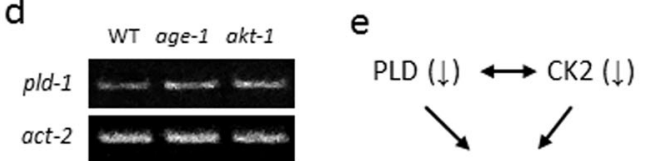

DAF-16/FoxO3a ( $\downarrow)$

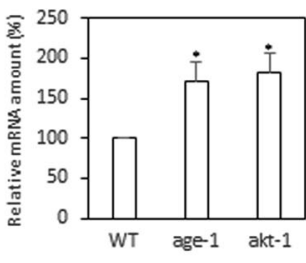

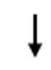

$\operatorname{ROS}(\uparrow)$

Aging/senescence $(\uparrow)$

Fig. 6 Effect of pld-1 RNAi on the lifespan of daf-16, age-1, and akt-1 single-mutant nematodes. Daf-16(mu86) (a), age-1(hx546) (b), and akt-1 (mg144) (c) single-mutant nematodes were fed on control RNAi or pld-1 RNAi plates under standard conditions ( $n=40$ per condition). Viability was scored as movement away from pick touch at the indicated days. Representative data from three independent RNAi experiments are shown. $\mathbf{d}$ Effect of age-1 (hx546) or akt-1 (mg 144) mutation on pld-1 expression. Lysates from nematodes at days 12 of adulthood were utilized in RT-PCR using specific pld-1 primers. PCR products were resolved on a 1.5\% agarose gel (upper panel). Representative data from three independent experiments are shown. act-2 mRNA served as the loading control. The graph shows the relative mRNA level of pld-1 (lower panel). Data are shown as the means \pm SEM. * $P<0.05$. e Possible model illustrating the role of DAF-16/FoxO3a in aging and senescence induced by PLD downregulation in C. elegans and human cells

1 and DAF-16 are positioned in the same pathway for lifespan control (Fig. 6a). Collectively, the present study demonstrated that DAF-16/FoxO3a plays a key role in PLD downregulation-mediated ROS accumulation in nematodes and human cells (Fig. 6d). In addition, the fact that pld-1 knockdown decreased the transcriptional activity of DAF-16 on the $m t l-1$ promoters while increasing the transcriptional activity on the $d o d-8$ promoter suggests that PLD downregulation may reduce stress resistance and increase fat accumulation through controlling DAF-16 activity in nematodes (Fig. 5a). MTL$1 /$ metallothionein is a heavy metal-binding protein that is involved in metal homeostasis ${ }^{26}$, and DOD-8/17 $\beta$-hydroxysteroid dehydrogenase is localized to lipid droplets, which are highly specialized for lipid storage ${ }^{27}$.

We previously showed that kin-10 (the ortholog of CK2 $\beta$ ) downregulation shortened nematode longevity and induced several age-related biomarkers (slowed motility, increased lipofuscin levels, decreased resistance to stress, and increased ROS levels) and that kin-10 knockdown decreased or increased the transcriptional activity of DAF-16 depending on the promoters of the target genes, demonstrating that CK2 regulates lifespan via the AGE-1AKT-1-DAF-16 pathway in nematodes ${ }^{28}$. Similar to results of a previous study $^{28}$, shortened lifespan and increased age-related biomarkers were also observed by pld-1 downregulation in this study. The present study showed that like CK2, PLD also regulates lifespan downstream of AGE-1 and AKT-1 (Fig. 6b, c) and upstream of DAF-16 in nematodes (Fig. 6a). All these data suggested the possibility that pld-1 knockdown may promote aging through a pathway identical to that of the kin-10 knockdown-mediated nematode aging. This possibility was also proposed by the fact that spermidine, which can act as a CK2 activator, successfully counteracted the lifespan shortening and age-related biomarkers mediated by pld-1 knockdown in nematodes (Fig. 4, Supplemental Figs. 2 and 3). This is consistent with the fact that PLD and CK2 positively regulate each other for the following reasons. First, CK2 downregulation, like PLD downregulation, induces cellular senescence via activating the ROS-p53-p21 Cip1/WAF1 pathway by downregulating FoxO3a in human cells ${ }^{29-32}$. Second, enhanced CK2 activity strongly represses PLD2 knockdown- 
mediated senescence in human cells, and vice versa ${ }^{14}$. Finally, PLD2 stimulates CK2 activity through activating protein kinase $\mathrm{C}^{18}$, whereas CK2 stimulates PLD activity via PLD phosphorylation in human cells ${ }^{33}$. Taken together, the previous and present studies strongly suggest that a positive regulation loop between PLD and CK2 may play a critical role in modulating longevity and senescence in nematode and human cells (Fig. 6e). A more complete understanding of the PLD-CK2-DAF-16/FoxO3a network will deliver an improved understanding of organism aging and cellular senescence.

\section{Acknowledgements}

This research was supported by the Basic Science Research Program through the National Research Foundation of Korea (NRF) funded by the Ministry of Science, ICT and Future Planning (NRF-2015R1A2A2A01004593) and Institute for Basic Science (IBS-R013-D1).

\section{Author details}

${ }^{1}$ School of Life Sciences, BK21 Plus KNU Creative BioResearch Group, College of Natural Sciences, Kyungpook National University, Daegu 41566, Republic of Korea. ${ }^{2}$ School of Life Sciences, College of Natural Sciences, Kyungpook National University, Daegu 41566, Republic of Korea. ${ }^{3}$ Center for Plant Aging Research, Institute for Basic Science, Daegu 42988, Republic of Korea

\section{Conflict of interest}

The authors declare that they have no conflict of interest.

\section{Publisher's note}

Springer Nature remains neutral with regard to jurisdictional claims in published maps and institutional affliations.

Supplementary information accompanies this paper at https://doi.org/ 10.1038/s12276-017-0015-8.

Received: 21 August 2017 Revised: 1 December 2017

Accepted: 5 December 2017.

Published online: 6 April 2018

\section{References}

1. Frohman, M. A. The phospholipase D superfamily as therapeutic targets. Trends Pharmacol. Sci. 36, 137-144 (2015).

2. Bruntz, R. C., Lindsley, C. W. \& Brown, H. A. Phospholipase D signaling pathways and phosphatidic acid as therapeutic targets in cancer. Pharmacol. Rev. 66, 1033-1079 (2014).

3. Brown, H. A., Thomas, P. G. \& Lindsley, C. W. Targeting phospholipase D in cancer, infection and neurodegenerative disorders. Nat. Rev. Drug Discov. 16, 351-367 (2017).

4. Kang, D. W., Choi, K. Y. \& Min, D. S. Functional regulation of phospholipase D expression in cancer and inflammation. J. Biol. Chem. 289, 22575-22582 (2014).

5. Raghu, P., Manifava, M., Coadwell, J. \& Ktistakis, N. T. Emerging findings from studies of phospholipase $D$ in model organisms (and a short update on phosphatidic acid effectors). Biochim. Biophys. Acta 1791, 889-897 (2009).

6. Liu, L. X. et al. High-throughput isolation of Caenorhabditis elegans deletion mutants. Genome Res. 9, 859-867 (1999).

7. Matthies, D. S., Fleming, P. A., Wilkes, D. M. \& Blakely, R. D. The Caenorhabditis elegans choline transporter $\mathrm{CHO}-1$ sustains acetylcholine synthesis and motor function in an activity-dependent manner. J. Neurosci. 26, 6200-6212 (2006).

8. Kenyon, C. J. The genetics of ageing. Nature 464, 504-512 (2010).
9. Altintas, O., Park, S. \& Lee, S. J. The role of insulin/IGF-1 signaling in the longevity of model invertebrates, C. elegans and D. melanogaster. BMB Rep. 49, 81-92 (2016).

10. Murphy, C. T. et al. Genes that act downstream of DAF-16 to influence the lifespan of Caenorhabditis elegans. Nature 424, 277-283 (2003).

11. Klotz, L. O. et al. Redox regulation of FoxO transcription factors. Redox. Biol. $\mathbf{6}$, 51-72 (2015).

12. Webb, L. M., Arnholt, A. T. \& Venable, M. E. Phospholipase D modulation by ceramide in senescence. Mol. Cell. Biochem. 337, 153-158 (2010).

13. Venable, M. E. \& Obeid, L. M. Phospholipase D in cellular senescence. Biochim. Biophys. Acta 1439, 291-298 (1999).

14. Lee, Y. H. \& Bae, Y. S. Phospholipase D2 downregulation induces cellular senescence through a reactive oxygen species-p53-p21 ${ }^{\text {Cip1/NAF1 }}$ pathway. FEBS Lett. 588, 3251-3258 (2014).

15. Lee, S. J., Hwang, A. B. \& Kenyon, C. Inhibition of respiration extends C. elegans life span via reactive oxygen species that increase HIIF-1 activity. Curr. Biol. 20, 2131-2136 (2010).

16. Hahm, J. H. et al. C. elegans maximum velocity correlates with healthspan and is maintained in worms with an insulin receptor mutation. Nat. Commun. $\mathbf{6}$, 8919 (2015).

17. Gerstbrein, B., Stamatas, G., Kollias, N. \& Driscoll, M. In vivo spectrofluorimetry reveals endogenous biomarkers that report healthspan and dietary restriction in Caenorhabditis elegans. Aging Cell 4, 127-137 (2005).

18. Lee, Y. H., Park, J. W. \& Bae, Y. S. Regulation of protein kinase CK2 catalytic activity by protein kinase C and phospholipase D2. Biochimie 121, 131-139 (2016).

19. Leroy, D., Heriche, J. K., Filhol, O., Chambaz, E. M. \& Cochet, C. Binding of polyamines to an autonomous domain of the regulatory subunit of protein kinase CK2 induces a conformational change in the holoenzyme. A proposed role for the kinase stimulation. J. Biol. Chem. 272, 20820-20827 (1997).

20. Shore, L. J., Soler, A. P. \& Gilmour, S. K. Ornithine decarboxylase expression leads to translocation and activation of protein kinase CK2 in vivo. J. Biol. Chem. 272, 12536-12543 (1997).

21. Zhang, P., Judy, M., Lee, S. J. \& Kenyon, C. Direct and indirect gene regulation by a life-extending FOXO protein in C. elegans: roles for GATA factors and lipid gene regulators. Cell Metab. 17, 85-100 (2013).

22. Sohal, R. S. \& Weindruch, R. Oxidative stress, caloric restriction, and aging. Science 273, 59-63 (1996).

23. Bokov, A., Chaudhuri, A. \& Richardson, A. The role of oxidative damage and stress in aging. Mech. Ageing Dev. 125, 811-826 (2004).

24. Ozden, $\mathrm{O}$. et al. Acetylation of $\mathrm{MnSOD}$ directs enzymatic activity responding to cellular nutrient status or oxidative stress. Aging (Albany NY) 3, 102-107 (2011).

25. Jeffery, J. \& Jörnvall, H. Sorbitol dehydrogenase. Adv. Enzymol. 61, 47-106 (1988).

26. Swindell, W. R. Metallothionein and the biology of aging. Ageing Res. Rev. 10, 132-145 (2011)

27. Su, W. et al. Comparative proteomic study reveals $17 \beta-H S D 13$ as a pathogenic protein in nonalcoholic fatty liver disease. Proc. Natl Acad. Sci. USA 111, 11437-11442 (2014)

28. Park, J. H. et al. Downregulation of protein kinase CK2 activity induces agerelated biomarkers in C. elegans. Oncotarget 8, 36950-36963 (2017).

29. Ryu, S. W. et al. Down-regulation of protein kinase CKII is associated with cellular senescence. FEBS Lett. 580, 988-994 (2006).

30. Kang, J. Y., Kim, J. J., Jang, S. Y. \& Bae, Y. S. The p53-p21 ${ }^{\text {cip } 1 \text { MAF1 }}$ pathway is necessary for cellular senescence induced by the inhibition of protein kinase CKII in human colon cancer cells. Mol. Cells 28, 489-494 (2009).

31. Jeon, S. M., Lee, S. J., Kwon, T. K., Kim, K. \& Bae, Y. S. NADPH oxidase is involved in protein kinase CKII down-regulation-mediated senescence through elevation of the level of reactive oxygen species in human colon cancer cells. FEBS Lett. 584, 3137-3142 (2010).

32. Park, S. Y. \& Bae, Y. S. Inactivation of the FoxO3a transcription factor is associated with the production of reactive oxygen species during protein kinase CK2 downregulation-mediated senescence in human colon cancer and breast cancer cells. Biochem. Biophys. Res. Commun. 478, 18-24 (2016).

33. Ahn, B. H., Min, G., Bae, Y. S., Bae, Y. S. \& Min, D. S. Phospholipase D is activated and phosphorylated by casein kinase-ll in human U87 astroglioma cells. Exp. Mol. Med. 38, 55-62 (2006). 